\title{
Optimal Uncoded Regeneration for Binary Antipodal Signaling
}

\author{
Ibrahim Abou-Faycal \\ Department of Electrical \& Computer Engineering \\ American University of Beirut \\ Beirut, Lebanon, 11072020 \\ Email: iaf@alum.mit.edu
}

\author{
Muriel Médard \\ Lab. for Information and Decision Systems \\ Massachusetts Institute of Technology \\ Cambridge, MA 02139 \\ Email: medard@mit.edu
}

\begin{abstract}
We derive, for a binary antipodal input signal, the optimal uncoded regenerator function when the channels at the ingress and at the egress of the regenerator are degraded by AWGN. We show that the optimal function is a Lambert $W$ function parametrized on the energies of the noises and the input. For comparison, we derive the performance of systems in which the regenerator uses a hard limiter or an amplifier.
\end{abstract}

\section{INTRODUCTION}

The problem we pose is simple and, we believe, fundamental. Given a binary antipodal input signal and a channel with band-limited additive white Gaussian noise (AWGN), what is the symbol-wise optimum processing that can be performed in-line to minimize the probability of error at the receiver. Figure 1 shows the system we consider. We assume perfect timing, as well as energy constraints at the transmitter and the processor.

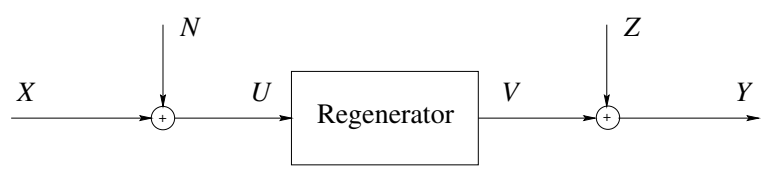

Fig. 1. System under consideration

Since we consider an uncoded system, degradations caused by the channel cannot be actually reversed. In this sense, uncoded regeneration is impossible, since the processing applied to attempt to remove the deleterious effects of the channel may itself introduce errors. Fundamentally, unknown random noise cannot be removed, as sometimes is assumed. The best we may hope for is to process the signal is such a way as to improve the bit error rate. Hence, it is more appropriate to speak of processing rather than regeneration. The term regeneration, however, is commonly used in the literature to describe in-line processing to improve end-to-end performance, as we discuss below. When discussing relevant results in the literature, we shall therefore adopt the common term of regeneration, but will prefer to speak of processing when discussing the function to be performed in the processor of Figure 1.

The problem of uncoded regeneration for binary, generally OOK, signals has been explored for many years. It was originally investigated for coaxial systems [1], and is particularly relevant in optical transmission systems. The problem of regenerators, sometimes called repeaters, is central to the issue of creating optical-electronic-optical (OEO) elements to counter the accumulation of noise in optical systems [2]. In optical transmission systems, the high transmission rates generally associated with optical transmission may render decoding/coding at the line rate overly onerous in terms of delay and processing.

The literature for regeneration of binary signals is rich. There is no single nomenclature for regeneration, but a fairly common usage is that of $1 \mathrm{R}$ (linear amplification), 2R (amplification and repeater) and $3 \mathrm{R}$ (amplification, repeater and retiming) [1], [3], [4]. The repeater is a non-linear function, most commonly a hard limiter. $2 \mathrm{R}$ systems are a common means of regeneration in optical systems [5]-[7] and many 3R systems have been demonstrated and proposed [8]-[10]. Unlike $3 \mathrm{R}$ systems, they do not require retiming and consideration of jitter issues.

The vast majority of the literature on regeneration is concerned with OOK signals, be they non-return to zero (NRZ) or return to zero (RZ) (solitons and signals of the same kin we subsume under ). Some authors have considered, either theoretically or experimentally, systems such as ternary signaling, quadrature phase-shift keying (QPSK) and frequency-shift keying (FSK).

Almost all of the body of literature dealing with regeneration discussed above assumes that the nonlinear function is a hard-limiter. The problem of observing through hard limiters noise [11], [12], noise-contaminated signals [13]-[15] and various sinusoids [16] is a classic problem in communication theory. More general non-linearities have been considered for phase-modulated signals [17], noise-contaminated signals [18] and Markov processes in general [19].

None of the the above work, however establish whether the commonly used hard limiter is indeed the best function to perform regeneration, or what other function may be optimal. Attempts at comparing the performance of different functions in regeneration have been limited. In [2], the authors consider three different non-linear functions in a cascade of repeaters or regenerators. The three different filters are chosen heuristically and their optimality is not considered.

In this work, we consider the optimal processing (regeneration) function for a binary antipodal signaling system with 
band-limited AWGN. We assume perfect synchronicity. Thus, we do not consider issues of sampling, such as arise in hardlimited systems [20].

In Section II, we present our channel model and the problem we investigate. In Section III and IV we analyze the performance of an amplifier and a hard decoder respectively. In Section $\mathrm{V}$, we design the optimal relay and analyze its performance. We show that the optimal relay consists of a Lambert function, whose parameters vary with the channel and signal characteristics. In particular, for low SNR regimes, the Lambert function resembles a hard limiter, and for high SNR regimes it resembles an amplifier. Our result are congruent with the extensive family of empirical observations in this area, which indicate that low SNR regimes call for hard limiters, whereas high SNR regimes are well served by amplifiers. In Section VI, we provide conclusions and directions for generalizing our results.

\section{Channel Model}

We consider the following discrete-time model for the relay channel

$$
Y=f(X+N)+Z,
$$

where $X$ is the channel input, $Y$ the output, and $N$ and $Z$ are independent Gaussian random variables with mean zero and variance $\sigma_{1}^{2}$ and $\sigma_{2}^{2}$ respectively. To accommodate for energy limitations we impose on the input $X$ an average power constraint: $\mathrm{E}\left[|X|^{2}\right] \leq P_{1}(\neq 0)$, as well as on the output of the relay: $\mathrm{E}\left[|f(X+N)|^{2}\right] \leq P_{2}(\neq 0)$. For notational convenience we define random variables $U=X+N$, and $V=f(U)$.

Furthermore, binary phase shift keying is assumed at the input, i.e. the input random variable $X$ is considered is

$$
X=\left\{\begin{aligned}
\sqrt{P_{1}}, & \text { with probability } 1 / 2 \\
-\sqrt{P_{1}}, & \text { with probability } 1 / 2 .
\end{aligned}\right.
$$

Since the system is uncoded, the receiver is assumed to perform a binary hypothesis test to decide which symbol was transmitted.

The objective of this study is to quantify the average probability of error of some of the relays suggested in the literature and design the optimal relay, that is, the one that minimizes this average probability of error.

Overall, in addition to designing the optimal relay, we consider two schemes:

1) Amplifying or scaling, in which case the function $f($. is linear. We will refer to this case as scheme (1).

2) Hard decision, where the output of the relay is a scaled hard decision on the input. We will refer to this case as scheme (2).

\section{AMPLIFIER}

In this section we conduct the probability of error analysis when an "amplifier" is used, or more precisely a linear function $f($.$) is used.$
To guarantee an average power constraint of $P_{2}$ at the output of the relay, the function $f($.$) is equal to$

$$
f(t)=\sqrt{\frac{P_{2}}{P_{1}+\sigma_{1}^{2}}} t,
$$

which yields an output

$$
\begin{aligned}
Y & =\sqrt{\frac{P_{2}}{P_{1}+\sigma_{1}^{2}}}(X+N)+Z \\
& =\sqrt{\frac{P_{2}}{P_{1}+\sigma_{1}^{2}}} X+\left(\sqrt{\frac{P_{2}}{P_{1}+\sigma_{1}^{2}}} N+Z\right) .
\end{aligned}
$$

Therefore, given $x$ was transmitted, the conditional probability density function of the output $Y$ is equal to

$$
p_{Y \mid X}(y \mid x) \sim \mathcal{N}\left(\sqrt{\frac{P_{2}}{P_{1}+\sigma_{1}^{2}}} X ; \frac{P_{2} \sigma_{1}^{2}}{P_{1}+\sigma_{1}^{2}}+\sigma_{2}^{2}\right),
$$

and the optimal decision rule is to decide on $\sqrt{P_{1}}$ if $y \geq 0$ and on $-\sqrt{P_{1}}$ otherwise.

Hence, the average probability of error of this scheme is

$$
\begin{aligned}
P_{e}(1)= & \frac{1}{2} \int_{-\infty}^{0} \mathcal{N}\left(u ; \sqrt{\frac{P_{2} P_{1}}{P_{1}+\sigma_{1}^{2}}}, \frac{P_{2} \sigma_{1}^{2}}{P_{1}+\sigma_{1}^{2}}+\sigma_{2}^{2}\right) d u \\
& +\frac{1}{2} \int_{0}^{\infty} \mathcal{N}\left(u ;-\sqrt{\frac{P_{2} P_{1}}{P_{1}+\sigma_{1}^{2}}}, \frac{P_{2} \sigma_{1}^{2}}{P_{1}+\sigma_{1}^{2}}+\sigma_{2}^{2}\right) d u \\
= & \int_{\sqrt{\frac{P_{2} P_{1}}{P_{1}+\sigma_{1}^{2}}}}^{\infty} \mathcal{N}\left(u ; 0, \frac{P_{2} \sigma_{1}^{2}}{P_{1}+\sigma_{1}^{2}}+\sigma_{2}^{2}\right) d u .
\end{aligned}
$$

\section{HARD DECISION}

In this scheme, the relay first performs hard decisions to decide using binary hypothesis testing on the input, then to satisfy the relay's output average energy constraint, $\sqrt{P_{2}}$ and $-\sqrt{P_{2}}$ are transmitted.

$$
\begin{aligned}
p_{Y \mid X}\left(y \mid \sqrt{P_{1}}\right)= & p_{Y, V \mid X}\left(y, \sqrt{P_{2}} \mid \sqrt{P_{1}}\right) \\
& +p_{Y, V \mid X}\left(y,-\sqrt{P_{2}} \mid \sqrt{P_{1}}\right) \\
= & p_{V \mid X}\left(\sqrt{P_{2}} \mid \sqrt{P_{1}}\right) p_{Y \mid V}\left(y \mid \sqrt{P_{2}}\right) \\
& +p_{V \mid X}\left(-\sqrt{P_{2}} \mid \sqrt{P_{1}}\right) p_{Y \mid V}\left(y \mid-\sqrt{P_{2}}\right) \\
= & p_{V \mid X}\left(\sqrt{P_{2}} \mid \sqrt{P_{1}}\right) \mathcal{N}\left(y ; \sqrt{P_{2}}, \sigma_{2}^{2}\right) \\
& +p_{V \mid X}\left(-\sqrt{P_{2}} \mid \sqrt{P_{1}}\right) \mathcal{N}\left(y ;-\sqrt{P_{2}}, \sigma_{2}^{2}\right) .
\end{aligned}
$$

The optimal decision rule at the receiver is to decide on $\sqrt{P_{1}}$ if $y \geq 0$ and on $-\sqrt{P_{1}}$ otherwise. Note that an error event occurs if either an error occurs at the relay and no error afterward, or no error occurs at the relay and then an error occurs at the receiver. The average probability of error is, after manipulation, given by

$$
\begin{aligned}
P_{e}(2)= & \int_{\sqrt{P_{1}}}^{\infty} \mathcal{N}\left(u ; 0, \sigma_{1}^{2}\right) d u \int_{-\sqrt{P_{2}}}^{\infty} \mathcal{N}\left(u ; 0, \sigma_{2}^{2}\right) d u \\
& +\int_{-\sqrt{P_{1}}}^{\infty} \mathcal{N}\left(u ; 0, \sigma_{1}^{2}\right) d u \int_{\sqrt{P_{2}}}^{\infty} \mathcal{N}\left(u ; 0, \sigma_{2}^{2}\right) d u .
\end{aligned}
$$




\section{OPTIMAL RELAY}

In this section we minimize the average probability of error over the set of sign-preserving symmetric functions $f($.$) . We$ first derive the average probability for any such function $f($.$) .$

Lemma 1: If $f($.$) is a sign preserving function then the$ optimal decision rule is to decide on $\sqrt{P_{1}}$ if $y \geq 0$ and on $-\sqrt{P_{1}}$ otherwise.

Proof: Note first that the probability law of the output given the input and a relay function $f($.$) is given by$

$$
\begin{aligned}
p_{Y \mid X}(y \mid x) & =\int_{-\infty}^{\infty} p_{U \mid X}(u \mid x) \mathcal{N}\left(y ; f(u), \sigma_{2}^{2}\right) d u \\
& =\int_{-\infty}^{\infty} \mathcal{N}\left(u ; x, \sigma_{1}^{2}\right) \mathcal{N}\left(y-f(u) ; 0, \sigma_{2}^{2}\right) d u .
\end{aligned}
$$

Therefore, the likelihood ratio is

$$
l=\frac{\int_{-\infty}^{\infty} \mathcal{N}\left(u ; \sqrt{P_{1}}, \sigma_{1}^{2}\right) \mathcal{N}\left(y-f(u) ; 0, \sigma_{2}^{2}\right) d u}{\int_{-\infty}^{\infty} \mathcal{N}\left(u ;-\sqrt{P_{1}}, \sigma_{1}^{2}\right) \mathcal{N}\left(y-f(u) ; 0, \sigma_{2}^{2}\right) d u} .
$$

The difference between the numerator and denominator may be simplified as follows:

$$
\begin{aligned}
& \int_{-\infty}^{\infty} \mathcal{N}\left(u ; \sqrt{P_{1}}, \sigma_{1}^{2}\right) \mathcal{N}\left(y-f(u) ; 0, \sigma_{2}^{2}\right) d u \\
- & \int_{-\infty}^{\infty} \mathcal{N}\left(u ;-\sqrt{P_{1}}, \sigma_{1}^{2}\right) \mathcal{N}\left(y-f(u) ; 0, \sigma_{2}^{2}\right) d u \\
= & \int_{0}^{\infty}\left[\mathcal{N}\left(u ; \sqrt{P_{1}}, \sigma_{1}^{2}\right)-\mathcal{N}\left(u ;-\sqrt{P_{1}}, \sigma_{1}^{2}\right)\right] \\
& {\left[\mathcal{N}\left(y-f(u) ; 0, \sigma_{2}^{2}\right)-\mathcal{N}\left(y-f(-u) ; 0, \sigma_{2}^{2}\right)\right] d u . }
\end{aligned}
$$

While the first difference is non-negative because $u \geq 0$, the second difference is also non-negative for $y \geq 0$ if $f(u)$ is non-negative in the range of the integration.

The average probability of error is hence given (after manipulation) by

$$
\begin{aligned}
P_{e}(f)= & \frac{1}{2} \int_{-\infty}^{\infty} \mathcal{N}\left(u ; \sqrt{P_{1}}, \sigma_{1}^{2}\right)\left[\int_{f(u)}^{\infty} \mathcal{N}\left(y ; 0, \sigma_{2}^{2}\right) d y\right] d u+ \\
& \frac{1}{2} \int_{-\infty}^{\infty} \mathcal{N}\left(u ;-\sqrt{P_{1}}, \sigma_{1}^{2}\right)\left[\int_{-f(u)}^{\infty} \mathcal{N}\left(y ; 0, \sigma_{2}^{2}\right) d y\right] d u
\end{aligned}
$$

If furthermore, $f($.$) is symmetric, i.e. f(-u)=-f(u)$, then by changing $u$ to $-u$ in the first term we obtain, after manipulation,

$$
P_{e}(f)=\int_{-\infty}^{\infty} \mathcal{N}\left(u ;-\sqrt{P_{1}}, \sigma_{1}^{2}\right)\left[\int_{-f(u)}^{\infty} \mathcal{N}\left(y ; 0, \sigma_{2}^{2}\right) d y\right] d u
$$

Next, we minimize the average probability of error over all suitable choices of $f($.$) . To prove achievability and existence$ of an optimal solution, we first establish the convexity of the optimization set and then we show that the objective function is convex. Finally we use functional analysis tools to find an optimal solution.

Lemma 2: The set $\Omega$ of sign-preserving, symmetric real functions with

$$
\int_{-\infty}^{\infty}\left[\mathcal{N}\left(u ;-\sqrt{P_{1}}, \sigma_{1}^{2}\right)+\mathcal{N}\left(u ; \sqrt{P_{1}}, \sigma_{1}^{2}\right)\right] f^{2}(u) d u \leq P_{2}
$$

is a convex set.

Proof: Let $f$ and $g$ be two functions in $\Omega$ and let $\lambda$ be a real non-negative number less or equal to one. Consider now the function $h(x)=\lambda f(x)+(1-\lambda) g(x)$. Since $\lambda$ and $(1-\lambda)$ are both non-negative, if $x<0$ then $f(x) \leq 0$ as well as $g(x) \leq 0$ which implies that $h(x) \leq 0$. Similarly for $x \geq 0$, $h(x) \geq 0$, which proves that $h$ is sign-preserving.

Furthermore,

$$
\begin{aligned}
h(-x) & =\lambda f(-x)+(1-\lambda) g(-x) \\
& =-\lambda f(x)-(1-\lambda) g(x)=-h(x),
\end{aligned}
$$

and hence $h$ is symmetric. Moreover, since $x^{2}$ is convex,

$$
\int_{-\infty}^{\infty}\left[\mathcal{N}\left(u ;-\sqrt{P_{1}}, \sigma_{1}^{2}\right)+\mathcal{N}\left(u ; \sqrt{P_{1}}, \sigma_{1}^{2}\right)\right] h^{2}(u) d u \leq P_{2}
$$

Lemma 3: The objective function (1) is convex.

Proof: Using equation (1), if a relay $f$ is used, the resulting average probability of error $P_{e}(f)$ is given by

$$
\begin{aligned}
P_{e}(f) & =\int_{-\infty}^{0} \mathcal{N}\left(u ; \sqrt{P_{1}}, \sigma_{1}^{2}\right) d u \\
+ & \int_{-\infty}^{0}\left[\mathcal{N}\left(u ;-\sqrt{P_{1}}, \sigma_{1}^{2}\right)-\mathcal{N}\left(u ; \sqrt{P_{1}}, \sigma_{1}^{2}\right)\right] \\
& {\left[\int_{-f(u)}^{\infty} \mathcal{N}\left(y ; 0, \sigma_{2}^{2}\right) d y\right] d u, }
\end{aligned}
$$

where we have omitted intermediary manipulation steps.

Note first that,

$$
\int_{x}^{\infty} \mathcal{N}\left(y ; 0, \sigma_{2}^{2}\right) d y
$$

is a strictly convex function of $x$ over the range $x \geq 0$. 
Hence, if $f \& g$ are in $\Omega$ and $\lambda \in[0,1]$ and $\gamma=1-\lambda$,

$$
\begin{aligned}
& P_{e}(\lambda f+\gamma g) \\
& \leq \lambda \int_{-\infty}^{0} \mathcal{N}\left(u ; \sqrt{P_{1}}, \sigma_{1}^{2}\right) d u \\
&+ \lambda \int_{-\infty}^{0}\left[\mathcal{N}\left(u ;-\sqrt{P_{1}}, \sigma_{1}^{2}\right)-\mathcal{N}\left(u ; \sqrt{P_{1}}, \sigma_{1}^{2}\right)\right] \\
& {\left[\int_{-f(u)}^{\infty} \mathcal{N}\left(y ; 0, \sigma_{2}^{2}\right) d y\right] d u } \\
&+\gamma \int_{-\infty}^{0} \mathcal{N}\left(u ; \sqrt{P_{1}}, \sigma_{1}^{2}\right) d u \\
&+\gamma \int_{-\infty}^{0}\left[\mathcal{N}\left(u ;-\sqrt{P_{1}}, \sigma_{1}^{2}\right)-\mathcal{N}\left(u ; \sqrt{P_{1}}, \sigma_{1}^{2}\right)\right] \\
& \quad\left[\int_{-g(u)}^{\infty} \mathcal{N}\left(y ; 0, \sigma_{2}^{2}\right) d y\right] d u \\
&=\lambda P_{e}(f)+\gamma P_{e}(g) .
\end{aligned}
$$

Finally, note that since function (2) is strictly convex, the objective function is strictly convex (when considering functions that differ on a set of non-zero measure.) Stated differently, the minimum probability achieving function $f$ is unique modulo differences on a set of zero measure.

To accommodate for the average power constraint at the output of the relay, we use the Lagrangian theorem and minimize the objective function

$$
\begin{gathered}
\mathrm{ob}(f)=P_{e}(f)+\lambda\left(\mathrm{E}\left[f^{2}(U)\right]-P_{2}\right) \\
=P_{e}(f)+\frac{\lambda}{2} \int_{-\infty}^{\infty}\left[\mathcal{N}\left(u ;-\sqrt{P_{1}}, \sigma_{1}^{2}\right)+\mathcal{N}\left(u ; \sqrt{P_{1}}, \sigma_{1}^{2}\right)\right] \\
{\left[f^{2}(u)-P_{2}\right] d u}
\end{gathered}
$$

where $\lambda$ is the Lagrange multiplier of the average power constraint $\mathrm{E}\left[f^{2}(U)\right] \leq P_{2}$, and satisfies the equation $\lambda\left(\mathrm{E}\left[f_{o}^{2}(U)\right]-P_{2}\right)=0$.

Theorem 5.1: The optimal relay function $f_{0}$ is given by

$f_{o}(u)= \begin{cases}\underbrace{}_{2} \sqrt{\sigma_{2}^{2} \text { LambertW }\left[2 \frac{\left(\tanh \left(u \frac{\sqrt{P_{1}}}{\sigma_{1}^{2}}\right)\right)^{2}}{\left(\lambda 2 \sigma_{2}^{2} \sqrt{\pi}\right)^{2}}\right]} & u \geq 0, \\ -f_{o}(-u) & u<0\end{cases}$

where $\lambda$ is such that

$$
\int_{-\infty}^{\infty}\left[\mathcal{N}\left(u ;-\sqrt{P_{1}}, \sigma_{1}^{2}\right)+\mathcal{N}\left(u ; \sqrt{P_{1}}, \sigma_{1}^{2}\right)\right] f_{o}^{2}(u) d u=2 P_{2}
$$

and LambertW $(x)$ is Lambert's W function analytic at 0 .

Proof: By convexity of the problem, a necessary and sufficient condition for the optimality of $f_{o}$ is

$$
\begin{aligned}
& \lim _{\epsilon \rightarrow 0^{+}} \frac{\mathrm{ob}\left((1-\epsilon) f_{o}+\epsilon g\right)-\mathrm{ob}\left(f_{o}\right)}{\epsilon} \\
= & \lim _{\epsilon \rightarrow 0^{+}} \frac{\mathrm{ob}\left(f_{o}+\epsilon\left(g-f_{o}\right)\right)-\mathrm{ob}\left(f_{o}\right)}{\epsilon} \geq 0,
\end{aligned}
$$

for all functions $g \in \Omega$. First, observe that

$$
\begin{aligned}
& \int_{-f_{o}-\epsilon\left(g-f_{o}\right)}^{\infty} h(x) d x-\int_{-f_{o}}^{\infty} h(x) d x \\
= & \int_{-f_{o}-\epsilon\left(g-f_{o}\right)}^{-f_{o}} h(x) d x=\epsilon h\left(-f_{o}\right)\left(g-f_{o}\right)+o\left(\epsilon^{2}\right)
\end{aligned}
$$

Also,

$$
\begin{aligned}
& \int_{-\infty}^{\infty} p_{U}(u)\left[f_{o}(u)+\epsilon\left(g(u)-f_{o}(u)\right)\right]^{2} d u-P_{2} \\
& -\left(\int_{-\infty}^{\infty} p_{U}(u) f_{o}^{2}(u) d u-P_{2}\right) \\
= & \int_{-\infty}^{\infty} p_{U}(u) \epsilon\left[g(u)-f_{o}(u)\right]\left[2 f_{o}(u)+\epsilon\left(g(u)-f_{o}(u)\right)\right] d u \\
= & \int_{-\infty}^{\infty} p_{U}(u) f_{o}(u) \epsilon\left(g(u)-f_{o}(u)\right) d u+o\left(\epsilon^{2}\right)
\end{aligned}
$$

In conclusion,

$$
\begin{gathered}
\lim _{\epsilon \rightarrow 0^{+}} \frac{\mathrm{ob}\left(f_{o}+\epsilon\left(g-f_{o}\right)\right)-\mathrm{ob}\left(f_{o}\right)}{\epsilon} \\
=\int_{-\infty}^{0}\left[\mathcal{N}\left(u ;-\sqrt{P_{1}}, \sigma_{1}^{2}\right)-\mathcal{N}\left(u ; \sqrt{P_{1}}, \sigma_{1}^{2}\right)\right] \\
\quad\left[\mathcal{N}\left(-f_{o}(u) ; 0, \sigma_{2}^{2}\right)\left(g(u)-f_{o}(u)\right)\right] d u \\
+\lambda \int_{-\infty}^{\infty}\left[\mathcal{N}\left(u ;-\sqrt{P_{1}}, \sigma_{1}^{2}\right)+\mathcal{N}\left(u ; \sqrt{P_{1}}, \sigma_{1}^{2}\right)\right] \\
{\left[f_{o}(u)\left(g(u)-f_{o}(u)\right)\right] d u .}
\end{gathered}
$$

Finally, the last step in the proof is to show that for $f_{o}(u)$ given in equation (3),

$$
\begin{aligned}
& {\left[\mathcal{N}\left(u ;-\sqrt{P_{1}}, \sigma_{1}^{2}\right)-\mathcal{N}\left(u ; \sqrt{P_{1}}, \sigma_{1}^{2}\right)\right] \mathcal{N}\left(-f_{o}(u) ; 0, \sigma_{2}^{2}\right)} \\
& +\lambda\left[\mathcal{N}\left(u ;-\sqrt{P_{1}}, \sigma_{1}^{2}\right)+\mathcal{N}\left(u ; \sqrt{P_{1}}, \sigma_{1}^{2}\right)\right] f_{o}(u)=0,
\end{aligned}
$$

for all negative values of $u$, or equivalently,

$$
\begin{aligned}
& \frac{\mathcal{N}\left(-f_{o}(u) ; 0, \sigma_{2}^{2}\right)}{-f_{o}(u)}=\frac{e^{-\left(-f_{o}(u) / \sqrt{2 \sigma_{2}^{2}}\right)^{2}}}{2 \sqrt{\pi} \sigma_{2}^{2}\left(-f_{o}(u) / \sqrt{2 \sigma_{2}^{2}}\right)} \\
& =\lambda \frac{\mathcal{N}\left(u ;-\sqrt{P_{1}}, \sigma_{1}^{2}\right)+\mathcal{N}\left(u ; \sqrt{P_{1}}, \sigma_{1}^{2}\right)}{\mathcal{N}\left(u ;-\sqrt{P_{1}}, \sigma_{1}^{2}\right)-\mathcal{N}\left(u ; \sqrt{P_{1}}, \sigma_{1}^{2}\right)},
\end{aligned}
$$

which can be further simplified to

$$
\frac{e^{-\left(-f_{o}(u) / \sqrt{2 \sigma_{2}^{2}}\right)^{2}}}{\left(-f_{o}(u) / \sqrt{2 \sigma_{2}^{2}}\right)}=\frac{\lambda 2 \sqrt{\pi} \sigma_{2}^{2}}{\tanh \left(\frac{-u \sqrt{P_{1}}}{\sigma_{1}^{2}}\right)}
$$

Which is satisfied due to the fact that LambertW $(x) e^{\text {LambertW }(x)}=x$.

Note that the optimal relay is not bounded to $\sqrt{P_{2}}$ like the hard limiter, and it does not increase linearly with $x$ as the amplifier. Also, the optimal relay is a function of all four parameters $P_{1}, P_{2}, \sigma_{1}^{2}$ and $\sigma_{2}^{2}$. Figure 2 shows a plot of the optimal relay for values of $P_{1}, P_{2}, \sigma_{1}^{2}$ and $\sigma_{2}^{2}$ all equal to 10 . As the value of noise variances decreases, the optimal relay looks closer like a hard limiter, and in the opposite case, it is more similar to an amplifier. 


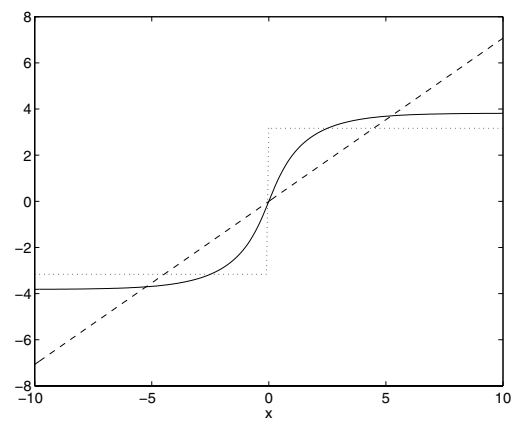

Fig. 2. Optimal Relay (solid line), Amplifier (dashed line) and Hard-Limiter (dotted)

Numerically, we computed the performance of an amplifier, a hard limiter, and the optimal relay. Figure 3 shows the relative difference between the probability of error with the optimal relay and that achieved when using a hard limiter. The powers $P_{1}$ and $P_{2}$ where taken to be 10 and the variances of $\sigma_{1}^{2}$ and $\sigma_{2}^{2}$ are represented on the axes.

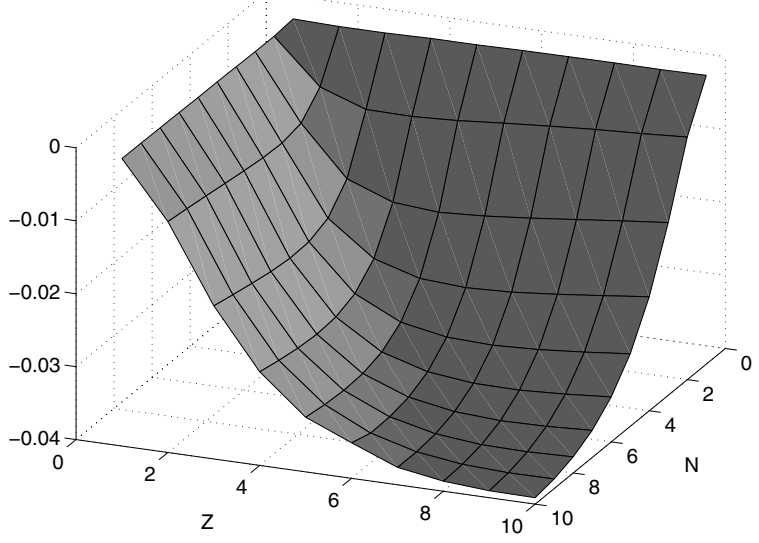

Fig. 3. Relative difference with respect to a hard-limiter

\section{CONCLUSIONS}

We have derived the general form of the function of the regenerator for binary antipodal signals in AWGN. The Lambert function we have derived resembles, in the high and low SNR cases, to a hard limiter and amplifier, respectively.

Our results may be readily extended to the case of OOK binary signaling. In optical transmission systems, OOK is the most common signaling scheme. The high transmission rates generally associated with optical transmission may render decoding/coding at the line rate overly onerous in terms of delay and processing. The receiver noise can be well modeled, at sufficiently high signal-to-noise ratio (SNR), as AWGN, without the need to consider Poisson models that are more relevant in the low-photon regime count. Other noise, such as amplitude-stimulated emission (ASE) from amplifiers such as Erbium-Doped Fiber Amplifiers (EDFAs) is also well modeled as AWGN. The problem we consider is also relevant to other transmission scenarios, such as low SNR communications in wireless settings where OOK is generally well-suited.

\section{REFERENCES}

[1] H. Marko, R. Weiß, and G. Binkert, "A digital hybrid transmission system for $280 \mathrm{Mbits} / \mathrm{s}$ and $560 \mathrm{Mbits} / \mathrm{s}$," in European Conference on Optical Communication (ECOC), vol. 4, 2001, pp. $574-575$.

[2] P. Öhlén and E. Berglind, "Noise accumulation and BER estimates in concatenated nonlinear optoelectronic repeaters," in IEEE Photonics Technology Letters, vol. 9, no. 7, July 1997, pp. 1011-1013.

[3] R. Barry and M. Médard, "Ber analysis of low-rate communications through a single electro-optic r2 nonlinear regenerator,' in Lasers and Electro-Optics (CLEO), 1998, pp. 455-456.

[4] J. Simon, L. Billes, A. Dupas, B. Kowalski, M. Henry, and B. Landousies, "All-optical regeneration," in European Conference on Optical Communication (ECOC), vol. 1, 1998, pp. 467-469.

[5] R. Gaudino, M. Len, G. Desa, M. Shell, and D. J. Blumenthal, "MOSAIC: a multiwavelength optical subcarrier multiplexed controlled network," jsac, vol. 16, 7, pp. $1270-1285,1998$.

[6] M. Matsumoto and O. Leclerc, "Analysis of 2R optical regenerator utilising self-phase modulation in highly nonlinear fibre," in Electronic Letters, vol. 38, no. 12, June 2002, pp. 576-577.

[7] J. D. Merlier, G. Morthier, T. V. Caenegem, R. Baefs, I. Moerman, and P. V. Daele, "Experimental demonstration of $15 \mathrm{~dB}$ extinction ratio improvement in a new 2R optical regenerator based on an MMI-SOA," in European Conference on Optical Communication (ECOC), vol. 4, 2001, pp. $574-575$.

[8] D. Chiaroni, B. Lavigne, A. Jourdan, L. Hamon, C. Janz, and M. Renaud, "New $10 \mathrm{Gbit} / \mathrm{s}$ 3R NRZ optical regenerative interface based on semiconductor optical amplifiers for all-optical networks," in European Conference on Optical Communication (ECOC), vol. 5, 1997, pp. 4145.

[9] O. Leclerc, P. Brindel, D. Rouvillain, B. Dany, E. Pincemin, E. Desurvire, C. Duchet, A. Shen, F. Blache, F. Devaux, A. Coquelin, M. Goix, S. Bouchoule, and P. Nouchi, "Dense WDM $(0.27 \mathrm{bit} / \mathrm{s} / \mathrm{Hz})$ $440 \mathrm{Gbit} / \mathrm{s}$ dispersion-managed transmission over $10000 \mathrm{~km}$ with in-line optical regeneration by channel pairs," in Electronic Letters, vol. 36, no. 4, February 2000, pp. 337-338.

[10] A. Kelly, I. Phillips, R. Manning, A. Ellis, D. Nesset, D. Moodie, and R. Kashyap, "Regeneration of optical frequency-shift-keying signal using self-pulsating and self-oscillating mechanisms in laser diodes," in Lasers and Electro-optics Society Annual Meeting (LEOS), vol. 2, 2000, pp. $440-441$.

[11] J. Mazo, R. Pawula, and S. Rice, "On a nonlinear problem involving RC noise," in IEEE Transactions on Information Theory, vol. IT-19, July 1973, pp. 404-410.

[12] L. Davisson and P. Papantoni-Kazakos, "On the distribution and moments of RC-filtered hard-limited RC-filtered white noise," in IEEE Transactions on Information Theory, vol. IT-19, July 1973, pp. 411414.

[13] O. Zeitouni, "On the filtering of noise-contaminated signals observed via hard limiters," in IEEE Transactions on Information Theory, vol. 34, no. 5, September 1988, pp. 1041-1048.

[14] J. Jones, "Hard-limiting of two signals in random noise," in IEEE Transactions on Information Theory, vol. IT-9, January 1963, pp. 34-42.

[15] P. Jain, "Limiting of signals in random noise," in IEEE Transactions on Information Theory, vol. IT-18, May 1972, pp. 332-340.

[16] W. Sollfrey, "Hard-limiting of three and four sinusoidal signals," in IEEE Transactions on Information Theory, vol. IT-15, January 1969, pp. 2-7.

[17] R. Lyons, "The effect of a bandpass nonlinearity on signal detectability," in IEEE Transactions on Communications, January 1973.

[18] N. Blachman, "The effect of a nonlineairty upon signals in the presence of noise," in IEEE Transactions on Communications, February 1973, pp. $152-154$.

[19] D. Darling and A. Siegert, "A systematic approach to a class of problems in the theory of noise and other random phenomena, parts i \& ii," in IRE Transactions on Information Theory, March 1957, pp. 32-43, see Also "part III" in the March 1958 issue, pp 4-14.

[20] I. Bar-David, "On the degradation of hard-limiting systems by sampling," in IEEE Transactions on Communications, vol. COM-25, no. 9, September 1977. 